\title{
Shifting the spotlight of attention: evidence for discrete computations in cognition
}

\section{Timothy J. Buschman* and Earl K. Miller}

Department of Brain and Cognitive Sciences and The Picower Institute for Learning and Memory, Massachusetts Institute of Technology, Cambridge, MA, USA

\section{Edited by:}

Thilo Womelsdorf, Robarts Research

Institute London, Canada

Reviewed by:

Juan R. Vidal, Universite Claude

Bernard, France

Tobias H. Donner, University of

Amsterdam, Netherlands

${ }^{*}$ Correspondence:

Timothy J. Buschman

46-6241, 77 Massachusetts Avenue

Cambridge, MA 02138 USA

e-mail: buschman@mit.edu
Our thoughts have a limited bandwidth; we can only fully process a few items in mind simultaneously. To compensate, the brain developed attention, the ability to select information relevant to the current task, while filtering out the rest. Therefore, by understanding the neural mechanisms of attention we hope to understand a core component of cognition. Here, we review our recent investigations of the neural mechanisms underlying the control of visual attention in frontal and parietal cortex. This includes the observation that the neural mechanisms that shift attention were synchronized to $25 \mathrm{~Hz}$ oscillatory brain rhythms, with each shift in attention falling within a single cycle of the oscillation. We generalize these findings to present a hypothesis that cognition relies on neural mechanisms that operate in discrete, periodic computations, as reflected in ongoing oscillations. We discuss the advantages of the model, experimental support, and make several testable hypotheses.

\section{Keywords: attention, cognition, synchrony, oscillations}

\section{INTRODUCTION}

Attention is the ability to select a particular stimulus for increased scrutiny. This ability is fundamental to cognition as it compensates for our limited capacity to process sensory inputs or hold items in mind. It does so by allowing the brain to flexibly represent certain items of interest at the expense of others. Sometimes those items are inherently salient and thus of potential interest (such as a looming object). This is often called "bottom-up", or stimulus-driven, attention. Alternatively, attention can be directed by "top-down" considerations such as internal valuations, regardless of an item's inherent saliency. Utilizing these internal goals is fundamental to going beyond simple stimulus-response behaviors and toward complex cognition.

Indeed, if we expand the role of attention from selecting external stimuli to also allowing the selection of internal representations (such as rules, action plans, stored memories, etc), attention is equivalent to cognitive control. Thus, by investigating the neural mechanisms of visual attention we hope to gain insight into the general mechanisms of cognitive control. The focus of this manuscript is to review the neural mechanisms underlying the control of attention (and then extend this toward cognition in general). Of particular interest will be the role of oscillations in neural computations and how this might inform our understanding of cognition. It is important to note that there has been extensive research into the effect of attention on the neural representation of selected stimuli (for reviews, see Desimone and Duncan, 1995; Corbetta and Shulman, 2002; Yantis and Serences, 2003; Maunsell and Treue, 2006) and although this is not the focus of this review we will rely on this literature in interpreting our findings.

\section{THE CONTROL OF VISUAL ATTENTION}

The most commonly used paradigm to study how attention is allocated is the visual search task (Treisman and Gelade, 1980; Duncan and Humphreys, 1989; Wolfe et al., 1989). Subjects (either monkey or humans) are asked to search a visual field for a particular target stimulus (see Figure 1A). How quickly the subject is able to do this depends on the relationship between the target and distracting visual elements. When distractors all differ from the target in a single dimension (Figure 1A, top row) the target will stand out, or "pop-out," from distracting stimuli, capturing attention automatically and leading to a very fast search time. What constitutes a pop-out dimension has been the subject of debate (for review, see Wolfe and Horowitz, 2004), but is generally viewed as the basic components of stimuli, such as color, orientation, shape, etc. Under these conditions not only can the subjects find the target quickly but the time to find the target is not strongly influenced by the number of distractors in the search array. In contrast, when the distractors differ from the target in more than one dimension, and do so independently from one another (Figure 1A, bottom row), the target no longer automatically grabs the subjects attention based on its inherent qualities, but rather internal signals (such as knowledge of the sought-after targets' attributes) must guide the search. This results in an overall slower search speed and, generally, the time to find the target is a function of the number of total items in the search array. In addition to the psychophysical work done in humans, behavioral differences between pop-out and search tasks have also been found in non-human primates (Iba and Sawaguchi, 2003; Buschman and Miller, 2007).

This dichotomy between fast and slow search reflects the mechanisms underlying the control attention: attention can either be captured by a stimulus based on its inherent physical properties or it can be directed toward a particular goal by an internal mechanism. This mirrors a major distinction in cognition, namely the distinction between automatic and controlled processing. The balance between them is important for cognition - external stimuli have intrinsic value based on their stimulus properties that can capture attention and behavior (i.e., fast things moving toward us should be salient). However, goal-directed behavior requires the ability to 
focus on particular stimuli or tasks despite the fact that they might not be the most salient. As we were interested in understanding how the brain balances these two competing processes we trained two monkeys to perform a visual search task. This allowed us to investigate the role of the frontal and parietal cortex in internal and external control of attention.

\section{FRONTAL CORTEX REFLECTS INTERNAL DIRECTION OF ATTENTION; PARIETAL CORTEX REFLECTS EXTERNAL CAPTURE OF ATTENTION}

Recent work has highlighted the importance of a frontal-parietal network in the control of attention (Corbetta et al., 1993, 1998; Coull et al., 1998). Human studies on the control of attention have isolated regions both in the parietal cortex (specifically within the intraparietal sulcus) and prefrontal cortex (including the human analog of the monkey frontal eye fields (FEF) in the precentral sulcus, Corbetta and Shulman, 2002). In humans, directing attention to a location (Corbetta et al., 1993; Coull and Nobre, 1998) or to a stimulus dimension (such as color or motion, Liu et al., 2003) leads to activation of regions within the precentral sulcus and intraparietal sulcus. There is even evidence that different "modes" of attentional selection (i.e., attending to location, color, or motion) may all be served by this same network (Liu et al., 2003; Yantis and Serences, 2003). Frontal-parietal networks are also engaged during visual search in humans (Corbetta et al., 1995; Donner et al., 2000, 2002; Nobre et al., 2002).

Neurophysiology has extended these results to single neurons in the analogous non-human primate regions in both parietal (specifically, the lateral intraparietal area, or LIP) and frontal cortex (both the lateral prefrontal cortex, IPFC and the FEF). Areas in the parietal cortex like area LIP are believed to carry a saliency map of the visual field and thus may play a leading role in the automatic allocation of attention during pop-out. Neurons in LIP reflect the attentional priority of stimuli in their receptive field (Bisley and Goldberg, 2003) and are known to respond transiently to flashed stimuli, which can automatically draw attention (Bisley and Goldberg, 2006). LIP neurons reflect the target location of a pop-out stimulus very quickly about $80 \mathrm{~ms}$ after the onset of the stimulus array (Ipata et al., 2006). The prefrontal cortex (including the FEF) is also critical for the allocation of attention. In addition to general deficits in behavioral control, patients with lesions to the PFC show specific deficits in visual search tasks (Eglin et al., 1991; Knight et al., 1995; Knight, 1997). In non-human primates, lPFC neurons carry information about the target location in visual popout (Hasegawa et al., 2000) and inactivating dlPFC with muscimol reduces their ability to do visual search tasks, but not detection tasks (Iba and Sawaguchi, 2003). FEF neurons have been shown to carry information about the locus of attention in both easy and difficult visual search tasks (Bichot and Schall, 1999).

Clearly, areas in both frontal and parietal cortex are involved in both types of attention (internal and external). However, in order to fully understand each areas respective role in controlling attention requires recording from all three regions simultaneously using multiple electrodes. Multiple-electrode recording is ideal for comparing different neuron populations because the neurons can be compared under identical conditions. The painstaking nature of the single-electrode approach means that different investigators tend to study neurons in different brain areas with different experimental paradigms in different animals with different training histories. All of this can affect neural activity and thus confounds comparisons across areas, potentially producing spurious differences and/or obscuring real ones (Miller and Wilson, 2008). Indeed, it is becoming increasingly apparent that attention (and cognitive control in general) does not exist in a single structure but instead relies on the interaction between many areas (as we will outline in greater detail below). Thus, without recording from multiple regions simultaneously it is impossible to determine the relative role of a brain area in a given function. For example, neural properties seen in a given area may have been computed elsewhere and have simply been inherited by the area in question, making it difficult to assign a region a particular function without knowing the properties of interacting brain areas.

Therefore, to directly test the relative roles of IPFC, FEF, and LIP in visual attention, we recorded from all three regions simultaneously while the monkeys performed a visual search task (alternating between the easy "pop-out" condition and the difficult "search" condition, Figure 1A). We found that when attention was automatically captured by a salient stimulus, neural signals reflected the direction of attention to that stimulus in area LIP with a shorter latency than in the IPFC and FEF. This suggests the bottom-up capture of attention by the salient target occurred in LIP before the frontal cortex. During the search condition, when attention must be directed by internal mechanisms, we found that the target location appeared in the frontal cortex (both IPFC and FEF) with a shorter latency than LIP (Buschman and Miller, 2007). Taken together, these results suggest that attention is captured by external stimuli in a bottom-up fashion; reflected in LIP (possibly as part of a saliency map) and fed-forward to frontal cortex (dashed arrows in Figure 1B). In contrast, the frontal cortex is the source of internal direction of attention, selecting particular stimuli based on internal goals and cognitive plans (solid arrows in Figure 1B). Similar results have recently been found in humans (Li et al., 2010). Having examined how the ultimate direction of attention is reflected in frontal and parietal cortex, we next examined the mechanisms of allocating attention when searching for the target.

\section{SERIAL DIRECTION OF THE SPOTLIGHT OF ATTENTION IN THE FRONTAL EYE FIELDS}

There is a general consensus that the automatic, bottom-up attentional selection by salient stimuli occurs through a parallel mechanism (Treisman and Gelade, 1980; Duncan and Humphreys, 1989; Wolfe et al., 1989; Reynolds et al., 1999; Itti and Koch, 2000). That is, as stimuli are processed along the visual cortex, they compete with one another for neural representation, with the most salient stimuli becoming the strongest represented because they garner more neural "energy" and thus win the competition. This may reach its culmination in LIP, with LIP carrying a map of the salience of the entire visual field. In contrast, the process of top-down, internal direction of attention during visual search is more controversial. Two major theories have been proposed. First, top-down attention could act by influencing the saliency values of all stimuli (i.e., increasing the "value" of all red stimuli and square stimuli when searching for a red square). These altered saliency values can then be compared, in parallel, as in the bottom-up condition. 


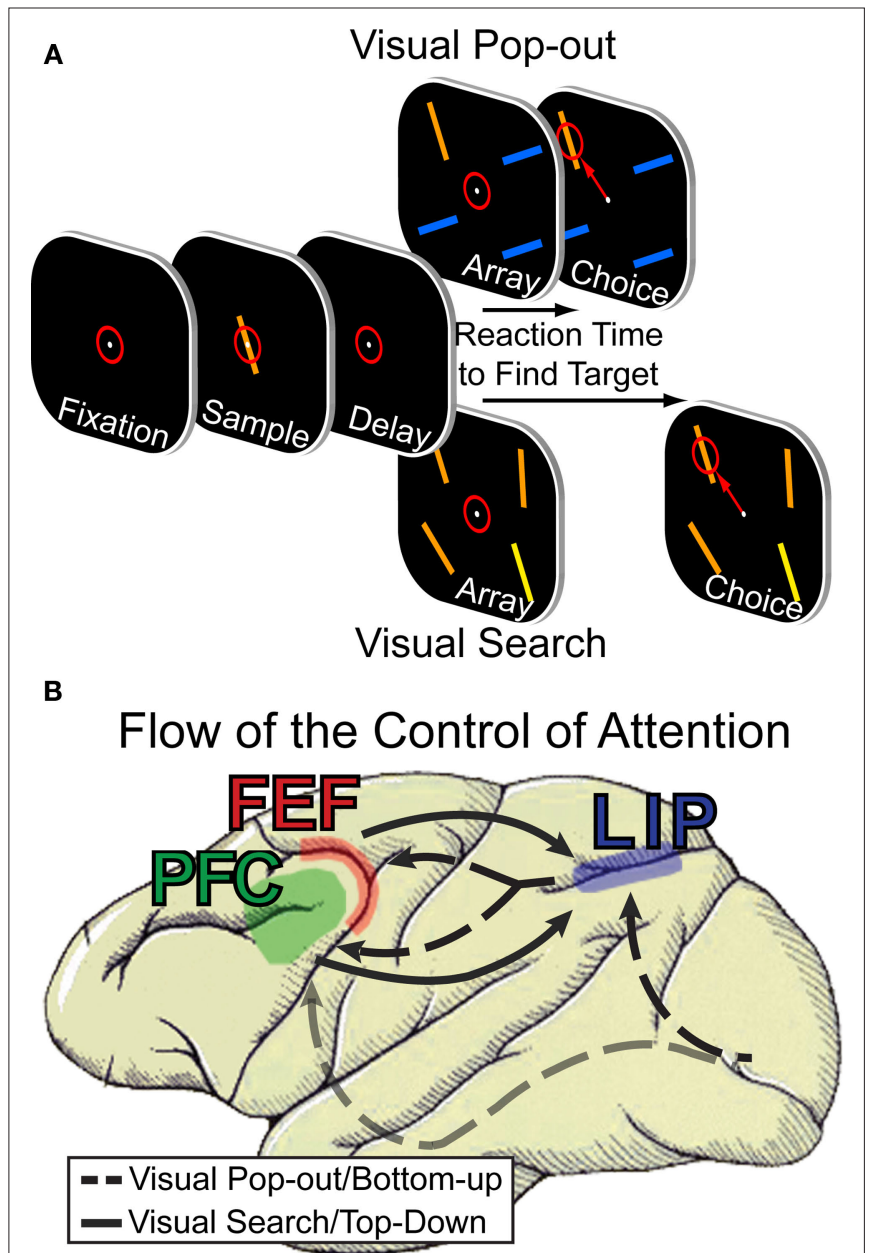

FIGURE 1 | (A) Outline of visual search task. Animals were required to search for a target stimulus in a visual array. (B) When attention was externally captured by the target (the pop-out condition), information about attention was found first in parietal cortex and then frontal cortex (dashed lines). In contrast, when attention was internally directed (the visual search condition), information was found first in frontal cortex and then posterior cortex (solid lines).

Alternatively, top-down signals could focus on individual stimuli, with each location attended in turn until the target is found. Both models explain psychophysical results well but make very different predictions about the nature of the top-down selection process. We examined our behavioral and neural data for evidence for either of these theories (Buschman and Miller, 2009).

Examining the animals' reaction time to find the target at each of the four possible locations revealed a striking pattern during visual search. Figure 2A shows the distribution of reaction times for a typical day - on this day the animal found the target the fastest when it was in the lower-right, slower when in the lower-left, slower still in the upper-left, and slowest in the upper-right. This suggests the animal tended to start its search process in the lowerright and continue in a clockwise fashion, allocating attention to each stimulus in turn. Across all of our recording sessions both animals seemed to have preferred starting points (although this did differ between animals) and both showed similar clockwise tendencies (see Buschman and Miller, 2009 for details). It is important

\section{A Search Time to Find Target at Each Location during Visual Search}

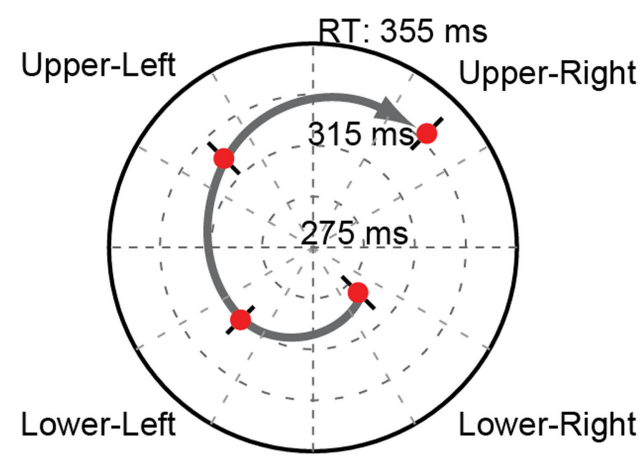

B

B Serial Activation of FEF Neurons during Visual Search

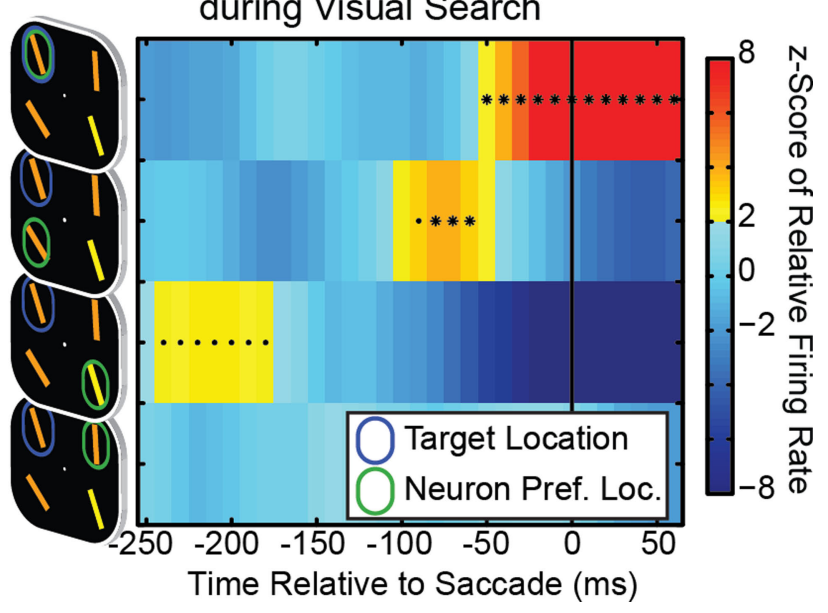

FIGURE 2 | (A) Example reaction time to find the target at each of the four possible locations. Reaction times suggest the animal preferred to start his search in the lower-right and then proceed clockwise. (B) Neural correlate of shifting spotlight of attention. Each row shows the activity of a neuron during trials with a specific offset between the target location (blue ellipse) and the neuron's preferred location (green ellipse). The activity of FEF neurons reflects the transient allocation of attention to each stimulus location in turn, proceeding clockwise toward the target location, as the trial progresses.

to note that we did not train monkeys to search in this clockwise serial fashion, they spontaneously adopted it (and it is an optimal serial search strategy).

Although this behavioral evidence for a serial search process is intriguing, it is not conclusive. As noted above, it could also reflect a parallel search process that is weighted toward certain visual field locations (i.e., some locations might be more salient than others). Direct evidence could come from examining neural activity for any evidence for serial, sequential activation of neurons consistent with a moving spotlight of attention. This is exactly what we found in the FEF (Figure 2B). FEF neurons with their preferred direction/receptive field at the target location responded just before the saccade was made (as expected, top row of Figure 2B). Interestingly, when the preferred direction of a FEF neuron was counter-clockwise to 
the target location we found a transient increase in neural activity consistent with the transient allocation of attention toward this location (second row down, Figure 2B). These neurons became active despite the fact that the eyes never moved toward that location (nor could we find any evidence for microsaccades in that direction, Buschman and Miller, 2009). We found even earlier activation for neurons with receptive fields at the previous counterclockwise "step" in the search process (Figure 2B, third row). We did not find this serial pattern of activation in $\mathrm{PFC}$ during search (instead IPFC neurons seem to be only active in response to finding the target at their preferred location), nor in parietal cortex. In fact, during search, LIP neurons did not significantly reflect the target location until after the saccade (Buschman and Miller, 2007). Interestingly, no area showed a serial pattern during visual pop-out; instead all three regions were only activated by the target in their preferred location, which is consistent with pop-out relying on parallel attentional processes.

We believe this is strong evidence that attention can be spatially directed to a particular location (by FEF) and repeated allocation of attention in this way can allow for a subject to search a visual array. Previous work by Tirin Moore and colleagues has shown that stimulating FEF neurons at subthreshold levels (i.e., levels that would not elicit a saccadic response) induces attention-like effects in V4 neurons with overlapping receptive fields (Moore and Armstrong, 2003) and that microstimulation in FEF will also boost the animal's behavioral discriminability at the target location (Moore and Fallah, 2001,2004). Further studies have shown that when directing attention to subparts of the receptive field the response of the V4 neuron will collapse around the selected region, in a manner similar to previous results (Reynolds et al., 1999; Armstrong et al., 2006). These results suggest that FEF may play a direct role in directing attention to specific portions of the receptive field and that this direction may come from the same regions that actually induce the eye to move, a model that our results support. In order to perform a complex search the animal can serially allocate attention to each stimulus in turn, as reflected in FEF neurons.

It is important to note that this does not exclude other forms of attention in visual search. Obviously, bottom-up signals are still valuable and can interact with top-down selection of stimulus attributes to generate a saliency map (Wolfe et al., 1989). The continuum of psychophysical results likely reflects the relative contribution of these more parallel mechanisms with the serial mechanisms shown here (Bichot et al., 2005; Bichot and Desimone, 2006).

\section{TASK SWITCHING AND INTER-AREAL SYNCHRONY}

Despite the differential roles played by areas in frontal and parietal cortex during visual pop-out and search it is clear that all three regions are involved in both of these tasks. This exemplifies a fundamental question in neuroscience-how do brain regions collaborate and communicate to produce complex behavior? One proposed solution is that altering the synchrony in neural activity between areas can change their effective connectivity, routing signals in a task-specific manner.

Synchrony can occur across several different scales. Synchronizing the activity of a local network of neurons all encoding a particular stimulus is thought to help propagate that representation to target regions. Coincidence of spikes from multiple neurons converging on a post-synaptic neuron has a super-additive effect (Aertsen et al., 1989; Usrey and Reid, 1999; Engel et al., 2001; Salinas and Sejnowski, 2001; Fries, 2005). Therefore, if sensory neurons tuned to the same stimulus synchronize their firing, that stimulus will be more strongly represented in downstream areas, as its impact on those "targets" is enhanced. In this fashion, local synchrony may help the brain to improve its signal to noise ratio while, at the same time, reduce the number of spikes needed to represent a stimulus (Aertsen et al., 1989; Tiesinga et al., 2002; Siegel and Konig, 2003). Because this is the goal of attention - to select specific stimuli for greater representation - it has led to the suggestion that attention might act by synchronizing stimulus representations in sensory cortex. Indeed, attention has been found to correlate with increased gamma and spiking synchrony within a region for visual (Fries et al., 2001; Womelsdorf et al., 2006) and somatosensory attention (Steinmetz et al., 2000; Bauer et al., 2006).

Synchrony between regions may regulate communication between brain areas. If neurons in one region are synchronous but their downstream targets are out of phase, then the benefits of local synchrony in the source will be lost. However, if brain areas oscillate in phase they are more likely to influence one another. This has led to the suggestion that inter-areal synchrony could be used to dynamically change the effective connection between regions (Bressler, 1996; Engel et al., 2001; Salinas and Sejnowski, 2001; Fries, 2005). Changing the relative phase alignment - or changing the ability of two areas to fall into an optimal coherence relationship - provides a mechanism by which the brain could flexibly gate the flow of information. Early support for this model comes from visual attention tasks: inter-areal coherence between "cognitive" regions (such as LIP or FEF) and sensory areas (such as MT or VT) has been found to increase with attention (Saalmann et al., 2007; Siegel et al., 2008; Gregoriou et al., 2009).

A further prediction of this model would be that the flexibility to switch between different behaviors will be reflected in changes in synchrony. To test this we examined synchrony between frontal and parietal cortex in our visual search paradigm. Although both frontal and parietal regions were involved in both top-down and bottom-up search, they seemed to make different contributions. Thus, there might be differences in the synchrony between the two regions during the two tasks that support their changing roles. This is exactly what we found: the frequency of coherence between prefrontal cortex and posterior parietal cortex depended on whether attention was top-down or bottom-up (see Figure 3, Buschman and Miller, 2007). When attention was automatically drawn to a target, coherence between parietal and frontal cortex was increased in the "gamma" band $(35-55 \mathrm{~Hz})$. However, when the animal internally directed its attention, coherence in the parietal-frontal network was increased in the "beta" band $(22-34 \mathrm{~Hz})$. Von Stein et al. (2000) found similar results in cats - synchrony between primary visual cortex and multimodal areas was stronger at lower frequencies when stimuli were associated with behavioral responses (suggesting a "top-down" component) and stronger at higher frequencies with behaviorally irrelevant novel stimuli (suggesting "bottom-up"). Changes in inter-areal synchrony for different behavioral tasks was also found by Pesaran et al. (2008), who showed an increase in synchrony between the parietal reach region 


\section{${ }^{A}$ Synchrony in Frontal-Parietal ${ }^{\text {B }}$ Difference in Frontal-Parietal Synchrony Network during Attention between Search and Pop-out}
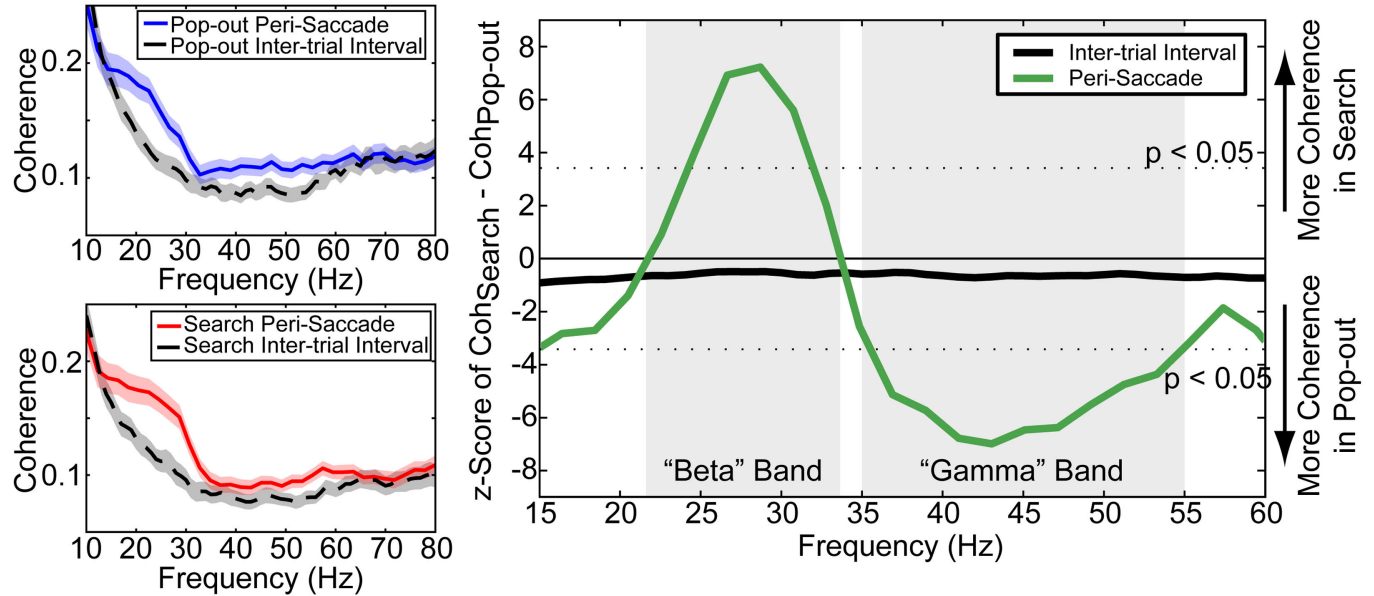

FIGURE 3 | (A) Synchrony between frontal and parietal cortex during visual pop-out (top) and visual search (bottom). Both tasks emphasize a "beta" band (18-34 Hz) and a "gamma" band $(35-55 \mathrm{~Hz})$ over baseline. However, there is a greater emphasis on gamma band activity during pop-out and beta band during search. (B) Direct comparison of synchrony between frontal and

and premotor cortex (frontal) around $15 \mathrm{~Hz}$ when the animal was allowed to move through a display freely compared to an instructed version of the same task.

\section{OSCILLATIONS PARSING COMPLEX COMPUTATIONS: CLOCKING THE SHIFTING SPOTLIGHT OF ATTENTION}

During our task, the role of an increase in gamma oscillations during visual pop-out (relative to visual search) seems to fit well with our general understanding of gamma oscillations - they exist to boost signal transduction between areas. As we know from the timing of neural activity, parietal cortex (LIP) is already encoding the location of the target and so this information should be passed forward into the frontal cortex for execution. Gamma-band synchrony might aid this process. In contrast, the role of "beta" band activity (approximately 18-34 Hz) that showed a stronger increase during top-down visual search is less clear. We noted, however, that this frequency band corresponded well with our behavioral and neural observations that the locus of attention shifted about every $40 \mathrm{~ms}$ ( $40 \mathrm{~ms}=25 \mathrm{~Hz})$. This led us to investigate the relationship between beta-band oscillations and the timing of these shifts of attention.

We took a decoding approach (for details, see Buschman and Miller, 2009). First, we used the classic neurophysiological approach of defining static time windows locked to an external event (in this case, the saccade). We empirically determined the optimal time windows that best captured the shifting spotlight of attention from the location just before the target to the target location. This turned out to be two time windows each about $33 \mathrm{~ms}$ wide and centered on 55 and 20 ms before the saccade, a result that agrees with our previous analyses showing a shift of attention about every $40 \mathrm{~ms}$ (see Figure 2). Next, we tested the hypothesis that shifts of attention were synchronized to beta band oscillations, by defining two parietal cortex across frequency. This comparison highlights the increase in synchrony between frontal and parietal cortex in the beta band during top-down, internally guided visual search. Likewise, an increase in gamma band synchrony is observed during bottom-up, externally driven pop-out.

analysis windows based on the one full oscillatory cycle just before the saccade (when attention was assumed to be at the target location) and the one full cycle before that (when attention was counterclockwise to the target location, see Figure 4A). The beta band oscillations were not strongly phase-locked to the saccade; there was variability in both the phase and frequency of the "beta" activity from trial to trial (four different trials are shown in Figure 4A with their "windows" of attention). If the shifts of attention were synchronized to beta band oscillation, then the oscillation-based windows should be better at decoding the location of attention than the static, saccade-based time windows (even though the static windows were optimized). Indeed, this is what we found, as shown in Figure 4B. The shaded red and green regions show the difference in normalized activity, a measure of information about whether the spotlight is counter-clockwise to or at the target location. This area is greater for the oscillation-based windows (solid lines) than for the static windows (dashed lines). Furthermore, in addition to significantly improving our ability to decode the locus of attention from the population (as shown in Figure 4B), using the dynamic, LFP-based, windows also improved decoding for the majority of individual neurons (Buschman and Miller, 2009). These results suggest that each cycle of the ongoing population oscillation reflects the allocation of attention to a particular stimulus location. The stimulus at that location is then presumably tested against the remembered target, and, if it does not match, the spotlight is shifted to the next stimulus location on the next cycle of oscillation.

\section{ARE COGNITIVE COMPUTATIONS DISCRETE?}

Our results provide evidence that shifting the spotlight of attention is discrete and timed by population oscillations: shifts tend to occur within a short window of time defined by the cycle of LFP oscillations. We propose to extend these results to all cognitive 
A Each Shift in Attention is Restricted to a Single Oscillatory Cycle

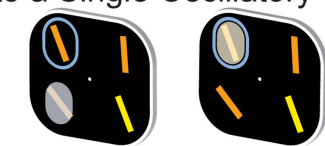

Dynamic "LFP"

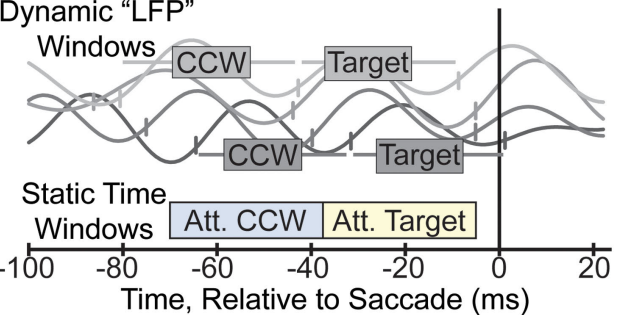

B

Direct Comparison of LFP \& Time Models: z-Score of Average Response

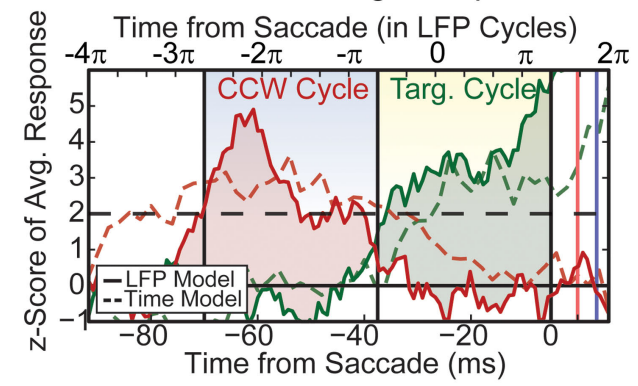

FIGURE 4 | (A) Diagram outlining how the shifting spotlight of attention is encapsulated by ongoing oscillations in the 18-34 Hz band. Circled object is the target, highlighted item is the currently attended location. Cycles of LFP oscillation were used to define periods of time when attention was at the CCW or Target location for each trial (show in gray boxes). This was compared to traditional static, time-based windows (shown along bottom). (B) Neural response to directing attention into a cell's preferred location, which can either be counter-clockwise (CCW) to the target location (red, second row of Figure 2) or at the target location (green, top row of Figure 2). The difference in response (shaded regions) reflects the information about the locus of attention. Information is greater when using the "dynamic" time windows defined by LFP oscillations (solid lines) compared to the static time-windows (dashed lines), suggesting each shift in attention occurs in a cycle of the beta oscillation.

computations. We review lines of evidence for this model, propose these windows may arise from feedback inhibition, and outline how they might be helpful for organizing neural computations for cognition.

It is well-established that the spiking activity of neurons is modulated by oscillations in vivo (e.g., Gray et al., 1989; Singer, 1999; Buzsaki and Draguhn, 2004) and artificially induced gamma oscillations have recently been shown to synchronize the spontaneous activity of local neurons (Cardin et al., 2009). This can be generalized by demonstrating that not only do population oscillations organize spiking per se but also the information encoded by spiking activity. For example, information for two objects held in working memory was found to be phase-locked to two different phases of a prominent $32 \mathrm{~Hz}$ oscillation in the PFC (Siegel et al., 2009). In this experiment, Siegel et al. trained monkeys to remember a sequence of two objects in short-term memory and found that information about the objects was pulsed at different phases of the underlying population oscillation, an explicit code of their remembered order. The phase-locking of spiking activity and information in the brain is predicted by our model - computations act upon and generate information and so if computations are temporally discrete and rhythmic then specific neural information should also be phase-locked to neural oscillations. In other words, our theory, which suggests neural computations are organized by the periods of population oscillations, is a superset of the theories suggesting spiking activity and information are phase-locked to oscillations.

The results of our visual search task also support a critical prediction of our model. We observed the parsing of a complex behavior (searching a visual field) into a simpler process (i.e., attend to a particular stimulus) that fell within an oscillatory cycle. In contrast to previous work where synchronized information is consistent from cycle to cycle (such as in the working memory task, Siegel et al., 2009), we found different information in each cycle (the focusing of attention on different locations) whereas the whole behavior (search of the visual field) was realized across cycles. Our behavioral results also support this model - we found that the reaction time to find the target was faster for trials with higher frequency oscillations (which would suggest the iterative computations were faster for that trial, Buschman and Miller, 2009). Psychophysical evidence for discrete computations has also been found both in visual perception (VanRullen et al., 2005, 2006) and in the allocation of attention (VanRullen et al., 2007). Furthermore, microsaccadic eye movements have been found to occur at specific phases of theta oscillations (Bosman et al., 2009), and the discrete and rhythmic nature of saccadic eye movements may be captured by ongoing low-frequency oscillations (Rajkai et al., 2008; Schroeder et al., 2010). Theta rhythms may also provide a similar functionality in the rodent hippocampus (Mehta et al., 2002; Buzsaki, 2005) - prospective and retrospective encoding in the hippocampus is known to be phase-locked to theta frequencies and the process of internally "looking" ahead or behind is discretized by the theta frequencies (Foster and Wilson, 2006; Davidson et al., 2009).

Local inhibitory feedback could provide the basis for these temporally discrete, rhythmic computations (Bartos et al., 2007). The typical wiring diagram of cortex consists of a local recurrent connection between excitatory and inhibitory neurons (Douglas and Martin, 2007). Theoretical and experimental work suggests that a strong excitatory drive activates inhibitory neurons which, in turn, suppress the local network (Siegel et al., 2000; Borgers and Kopell, 2003; Whittington and Traub, 2003; Hasenstaub et al., 2005; Cardin et al., 2009). These excitatory-inhibitory cycles may act to restrict neural processing to a window of time. In effect, it discretizes the computation, separating it into distinct epochs. Repeating these local excitatory-inhibitory cycles would result in the oscillations observed in the brain (Borgers and Kopell, 2005).

But what purpose might discrete computations serve? First, limiting neural computations to short bursts of time would ensure that informative spikes occur with the temporal precision both necessary for integration by downstream neurons and for spiketiming dependent plasticity (Lee et al., 2009). Second, it would act to stabilize and organize the network and its computations. Periods of inhibition may act to "reset" the network to a base state, effectively limiting the number of states that neurons could obtain. 
Returning to a stable state would provide a consistent base from which further processing could occur, ensuring that computations are reliable from moment to moment.

Restricting computations to discrete windows of time would also allow for easier coordination of processing within and between regions. Very simply, pulsing computations would allow the brain to better organize both the inputs and outputs to a computation. It provides a specific moment at which information must be available for computation in a specific region. On the other end, it also specifies a moment when the outcome of the computation is available. This provides a mechanism for ordering computations across brain regions - synchronization between areas would allow the outputs from one region to arrive at a target region in time to become inputs for the target's computation. Of course, organizing this activity between regions would require the synchronization of these regions and thus altering synchrony would still be a way to sculpt the flow of information (Fries, 2005; Haider and McCormick, 2009). Similarly, different frequency bands might still be used as different "carrier frequencies" with local computations carried by local, fast, oscillations while more complex functions require more distributed processing at lower frequencies (Varela et al., 2001). Coupling between frequencies could allow for multiplexing of information and computations (Lisman and Idiart, 1995; Lakatos et al., 2008; Sirota et al., 2008), as has been found during working memory (Canolty et al., 2006; Axmacher et al., 2010), learning (Tort et al., 2009), and reward processing (Cohen et al., 2009a,b). Finally, discrete computations may also allow the brain to actively sample the external world at a natural rhythm - there is intriguing data suggesting that rhythmic structure in the external world may be captured by internal oscillations (Lakatos et al., 2008; Schroeder and Lakatos, 2009). Although our own results are during a cognitive task, these results suggest our model may extend to sensory processing.

Our model makes several predictions. First, we expect to see more experimental evidence for neural computations being encapsulated by oscillations as researchers begin to look for them. Specifically, we would expect to find the complex computations underlying cognition to be broken down into simpler operations (each of which might be locally organized by different regions). We do want to note that our model does not require all computations in the brain to be discrete. For example, early stage stimulus processing and motor control systems may benefit from being continuous in nature, while the integrative nature of complex behaviors may require the pulsing of computations. The theory that oscillations reflect discrete computations also argues against any specific computational function for the oscillation itself (i.e., gamma oscillations are not always induced when boosting a stimulus representation in attention, as was recently shown in V1, Siegel et al., 2008; Chalk et al., 2010).

Finally, our model predicts the algorithms used by neural networks in the brain will be iterative or recursive in nature. This is due to the fact that the computation underlying a cognitive process

\section{REFERENCES}

Aertsen, A. M., Gerstein, G. L., Habib, M. K., and Palm, G. (1989). Dynamics of neuronal firing correlation: modulation of "effective connectivity". J. Neurophysiol. 61, 900-917.
Armstrong, K. M., Fitzgerald, J. K., and Moore, T. (2006). Changes in visual receptive fields with microstimulation of frontal cortex. Neuron 50, 791-798.

Axmacher, N., Henseler, M. M., Jensen, O., Weinreich, I., Elger, C. E., and Fell, J.

is broken down into smaller steps, with each simpler computation occurring in temporally distinct windows. Therefore, evolving the simpler computations into a complex cognitive behavior requires recurrent connections, feeding back the previous results from a "basic" process into the next iteration. This is of interest for neuroscientists attempting to understand the computational mechanisms of the brain - we would suggest that when one attempts to write down the algorithm, or process, that the brain takes in computing a behavior, that this algorithm should be recursive or iterative in nature. General models of how recursive functions might exist in the brain have been well developed (Rao, 2004) and specific recursive algorithms are even being proposed for specific cognitive behaviors, such as decision-making under uncertainty using the classic paradigm of integrating noisy motion stimuli (Wang, 2008).

\section{CONCLUSION}

Attention allows the brain to dynamically select pertinent information relevant to the current behavior, while ignoring irrelevant distractors. Understanding the neural mechanisms underlying its control will yield important clues to how the brain directs behavior. We found that information about where attention was allocated can either originate in posterior cortex (when grabbed by external cues) or frontal cortex (when being internally directed). Furthermore, we found both neural and behavioral evidence for a serial mechanism underlying visual search while results from visual pop-out are more consistent with a parallel mechanism.

These differences in neural mechanisms were also reflected in the synchronization of these regions. Visual pop-out showed greater high-frequency, gamma-band, synchrony, likely reflecting the passing of information from LIP into IPFC and FEF for action. In contrast, visual search showed enhanced synchrony in the beta-band, whose oscillations were found to capture the process of serially shifting attention.

These results, along with a diversity of data from other labs, lead us to propose that the neural computations underlying cognition are temporally discrete. These pulses are due to local feedback inhibition and can be concatenated to form oscillations. We believe these discrete computations would be more stable and easier to organize than a continuously evolving system, making it easier to integrate processing across several brain regions. Although this model is still developing, we believe there is a growing body of evidence supporting the idea that cognitive computations are temporally discrete as reflected in observed neural oscillations.

\section{ACKNOWLEDGMENTS}

We thank S. Henrickson and M. Wicherski for comments on the manuscript; J. Roy and M. Siegel for insightful discussions and for comments on the manuscript.

(2010). Cross-frequency coupling supports multi-item working memory in the human hippocampus. Proc. Natl. Acad. Sci. U.S.A. 107, 3228-3233.

Bartos, M., Vida, I., and Jonas, P. (2007). Synaptic mechanisms of synchronized gamma oscillations in inhibitory interneuron networks. Nat. Rev. 8, 45-56.

Bauer, M., Oostenveld, R., Peeters, M., and Fries, P. (2006). Tactile spatial attention enhances gamma-band activity 
in somatosensory cortex and reduces low-frequency activity in parieto-occipital areas. J. Neurosci. 26, 490-501.

Bichot, N. P., and Desimone, R. (2006). Finding a face in the crowd: parallel and serial neural mechanisms of visual selection. Prog. Brain Res. 155, 147-156.

Bichot, N.P., Rossi, A. F., and Desimone, R. (2005). Parallel and serial neural mechanisms for visual search in macaque area V4. Science 308, 529-534.

Bichot, N. P., and Schall, J. D. (1999). Saccade target selection in macaque during feature and conjunction visual search. Vis. Neurosci. 16, 81-89.

Bisley, J. W., and Goldberg, M. E. (2003). Neuronal activity in the lateral intraparietal area and spatial attention. Science 299, 81-86.

Bisley, J. W., and Goldberg, M. E. (2006). Neural correlates of attention and distractibility in the lateral intraparietal area. J. Neurophysiol. 95, 1696-1717.

Borgers, C., and Kopell, N. (2003). Synchronization in networks of excitatory and inhibitory neurons with sparse, random connectivity. Neural. Comput. 15, 509-538.

Borgers, C., and Kopell, N. (2005). Effects of noisy drive on rhythms in networks of excitatory and inhibitory neurons. Neural. Comput. 17, 557-608.

Bosman, C.A., Womelsdorf, T., Desimone, R., and Fries, P. (2009). A microsaccadic rhythm modulates gammaband synchronization and behavior. J. Neurosci. 29, 9471-9480.

Bressler, S. L. (1996). Interareal synchronization in the visual cortex. Behav. Brain Res. 76, 37-49.

Buschman, T. J., and Miller, E. K. (2007). Top-down versus bottom-up control of attention in the prefrontal and posterior parietal cortices. Science 315, 1860-1862.

Buschman, T. J., and Miller, E. K. (2009). Serial, covert shifts of attention during visual search are reflected by the frontal eye fields and correlated with population oscillations. Neuron 63 , 386-396.

Buzsaki, G. (2005). Theta rhythm of navigation: link between path integration and landmark navigation, episodic and semantic memory. Hippocampus $15,827-840$.

Buzsaki, G., and Draguhn, A. (2004). Neuronal oscillations in cortical networks. Science 304, 1926-1929.

Canolty, R. T., Edwards, E., Dalal, S. S., Soltani, M., Nagarajan, S. S., Kirsch, H. E., Berger, M. S., Barbaro, N. M., and Knight, R. T. (2006). High gamma power is phase-locked to theta oscillations in human neocortex. Science 313, 1626-1628.
Cardin, J. A., Carlen, M., Meletis, K. Knoblich, U., Zhang, F., Deisseroth, K., Tsai, L. H., and Moore, C. I. (2009). Driving fast-spiking cells induces gamma rhythm and controls sensory responses. Nature 459, 663-667.

Chalk, M., Herrero, J. L., Gieselmann, M. A., Delicato, L. S., Gotthardt, S., and Thiele, A. (2010). Attention reduces stimulus-driven gamma frequency oscillations and spike field coherence in V1. Neuron 66, 114-125.

Cohen, M. X., Axmacher, N., Lenartz, D. Elger, C. E., Sturm, V., and Schlaepfer, T. E. (2009a). Good vibrations: crossfrequency coupling in the human nucleus accumbens during reward processing. J. Cogn. Neurosci. 21 , 875-889.

Cohen, M. X., Elger, C. E., and Fell, J. (2009b). Oscillatory activity and phase-amplitude coupling in the human medial frontal cortex during decision making. J. Cogn. Neurosci. 21, 390-402.

Corbetta, M.,Akbudak, E., Conturo, T.E., Snyder, A. Z., Ollinger, J. M., Drury, H. A., Linenweber, M. R., Petersen, S. E., Raichle, M. E., Van Essen, D. C., and Shulman, G. L. (1998). A common network of functional areas for attention and eye movements. Neuron 21, 761-773.

Corbetta, M., Miezin, F. M., Shulman, G. L., and Petersen, S. E. (1993). A PET study of visuospatial attention. J. Neurosci. 13, 1202-1226.

Corbetta, M., and Shulman, G. L. (2002). Control of goal-directed and stimulusdriven attention in the brain. Nat. Rev 3, 201-215.

Corbetta, M., Shulman, G. L., Miezin, F. M., and Petersen, S. E. (1995). Superior parietal cortex activation during spatial attention shifts and visual feature conjunction. Science 270, 802-805.

Coull, J. T., Frackowiak, R. S., and Frith, C. D. (1998). Monitoring for target objects: activation of right frontal and parietal cortices with increasing time on task. Neuropsychologia 36, 1325-1334.

Coull, J. T., and Nobre, A. C. (1998). Where and when to pay attention: the neural systems for directing attention to spatial locations and to time intervals as revealed by both PET and fMRI. J. Neurosci. 18, 7426-7435.

Davidson, T. J., Kloosterman, F., and Wilson, M. A. (2009). Hippocampal replay of extended experience. Neuron 63, 497-507.

Desimone, R., and Duncan, J. (1995). Neural mechanisms of selective visual attention. Annu. Rev. Neurosci. 18, 193-222.

Donner, T., Kettermann, A., Diesch, E., Ostendorf, F., Villringer, A., and
Brandt, S. A. (2000). Involvement of the human frontal eye field and multiple parietal areas in covert visual selection during conjunction search. Eur. J. Neurosci. 12, 3407-3414.

Donner, T. H., Kettermann, A., Diesch, E., Ostendorf, F., Villringer, A., and Brandt, S. A. (2002). Visual feature and conjunction searches of equa difficulty engage only partially overlapping frontoparietal networks. Neuroimage 15, 16-25.

Douglas, R. J., and Martin, K. A. (2007). Mapping the matrix: the ways of neocortex. Neuron 56, 226-238.

Duncan, J., and Humphreys, G. W. (1989) Visual search and stimulus similarity. Psychol. Rev. 96, 433-458.

Eglin, M., Robertson, L. C., and Knight, R. T. (1991). Cortical substrates supporting visual search in humans. Cerebral Cortex 1, 262-272.

Engel, A. K., Fries, P., and Singer, W (2001). Dynamic predictions: oscillations and synchrony in top-down processing. Nat. Rev. 2, 704-716.

Foster, D. J., and Wilson, M. A. (2006) Reverse replay of behavioural sequences in hippocampal place cells during the awake state. Nature 440 680-683.

Fries, P. (2005). A mechanism for cognitive dynamics: neuronal communication through neuronal coherence. Trends Cogn. Sci. 9, 474-480.

Fries, P., Reynolds, J. H., Rorie, A. E., and Desimone, R. (2001). Modulation of oscillatory neuronal synchronization by selective visual attention. Science 291, 1560-1563.

Gray, C. M., Konig, P., Engel, A. K., and Singer, W. (1989). Oscillatory responses in cat visual cortex exhibit inter-columnar synchronization which reflects global stimulus properties. Nature 338, 334-337.

Gregoriou, G. G., Gotts, S. J., Zhou, H., and Desimone, R. (2009). High-frequency, long-range coupling between prefrontal and visual cortex during attention. Science 324, 1207-1210.

Haider, B., and McCormick, D. A. (2009) Rapid neocortical dynamics: cellular and network mechanisms. Neuron 62, 171-189.

Hasegawa, R. P., Matsumoto, M., and Mikami,A. (2000). Search target selection in monkey prefrontal cortex. $J$. Neurophysiol. 84, 1692-1696.

Hasenstaub, A., Shu, Y., Haider, B. Kraushaar, U., Duque, A., and McCormick, D. A. (2005). Inhibitory postsynaptic potentials carry synchronized frequency information in active cortical networks. Neuron 47 , 423-435.

Iba, M., and Sawaguchi, T. (2003). Involvement of the dorsolateral pre- frontal cortex of monkeys in visuospatial target selection. J. Neurophysiol.89, 587-599.

Ipata, A. E., Gee, A. L., Gottlieb, J., Bisley, J. W., and Goldberg, M. E. (2006). LIP responses to a popout stimulus are reduced if it is overtly ignored. Nat. Neurosci. 9, 1071-1076.

Itti, L., and Koch, C. (2000). A saliencybased search mechanism for overt and covert shifts of visual attention. Vision Res. 40, 1489-1506.

Knight, R. T. (1997). Distributed cortical network for visual attention. J. Cogn. Neurosci. 9, 75-91.

Knight, R. T., Grabowecky, M. F., and Scabini, D. (1995). Role of human prefrontal cortex in attention control. Adv. Neurol. 66, 21-34; discussion 34-26.

Lakatos, P., Karmos, G., Mehta, A. D., Ulbert, I., and Schroeder, C. E. (2008). Entrainment of neuronal oscillations as a mechanism of attentional selection. Science 320, 110-113.

Lee, S., Sen, K., and Kopell, N. (2009). Cortical gamma rhythms modulate NMDAR-mediated spike timing dependent plasticity in a biophysical model.PLoS Comput. Biol. 5, e1000602. doi: 10.1371/journal.pcbi.1000602.

Li, L., Gratton, C., Yao, D., and Knight, R. T. (2010). Role of frontal and parietal cortices in the control of bottom-up and top-down attention in humans. Brain Res. 1344, 173-184.

Lisman, J. E., and Idiart, M. A. (1995). Storage of $7+/-2$ short-term memories in oscillatory subcycles. Science 267, 1512-1515.

Liu, T., Slotnick, S. D., Serences, J. T., and Yantis, S. (2003). Cortical mechanisms of feature-based attentional control. Cereb. Cortex 13, 1334-1343.

Maunsell, J. H., and Treue, S. (2006). Feature-based attention in visual cortex. Trends Neurosci. 29, 317-322.

Mehta, M. R., Lee, A. K., and Wilson, M.A. (2002). Role of experience and oscillations in transforming a rate code into a temporal code. Nature 417, 741-746.

Miller, E. K., and Wilson, M.A. (2008). All my circuits: using multiple electrodes to understand functioning neural networks. Neuron 60, 483-488.

Moore, T., and Armstrong, K. M. (2003). Selective gating of visual signals by microstimulation of frontal cortex. Nature 421, 370-373.

Moore, T., and Fallah, M. (2001). Control of eye movements and spatial attention. Proc. Natl. Acad. Sci. U.S.A. 98, 1273-1276.

Moore, T., and Fallah, M. (2004). Microstimulation of the frontal eye field and its effects on covert spatial attention. J. Neurophysiol. 91, 152-162. 
Nobre, A. C., Sebestyen, G. N., Gitelman, D. R., Frith, C. D., and Mesulam, M. M. (2002). Filtering of distractors during visual search studied by positron emission tomography. Neuroimage 16, 968-976.

Pesaran, B., Nelson, M. J., and Andersen, R. A. (2008). Free choice activates a decision circuit between frontal and parietal cortex. Nature 453, 406-409.

Rajkai,C., Lakatos, P., Chen, C. M.,Pincze, Z., Karmos, G., and Schroeder, C. E. (2008). Transient cortical excitation at the onset of visual fixation. Cereb. Cortex 18, 200-209.

Rao, R. P. (2004). Bayesian computation in recurrent neural circuits. Neural. Comput. 16, 1-38.

Reynolds,J.H.,Chelazzi,L., and Desimone, R. (1999). Competitive mechanisms subserve attention in macaque areas $\mathrm{V} 2$ and V4. J. Neurosci. 19, 1736-1753.

Saalmann, Y. B., Pigarev, I. N., and Vidyasagar, T. R. (2007). Neural mechanisms of visual attention: how top-down feedback highlights relevant locations. Science 316, 1612-1615.

Salinas, E., and Sejnowski, T. J. (2001). Correlated neuronal activity and the flow of neural information. Nat. Rev. 2, 539-550.

Schroeder, C. E., and Lakatos, P. (2009). Low-frequency neuronal oscillations as instruments of sensory selection. Trends Neurosci. 32, 9-18.

Schroeder, C. E., Wilson, D. A., Radman, T., Scharfman, H., and Lakatos, P. (2010). Dynamics of active sensing and perceptual selection. Curr. Opin. Neurobiol. 20, 172-176.
Siegel, M., Donner, T. H., Oostenveld, R., Fries,P., and Engel,A.K. (2008). Neuronal synchronization along the dorsal visual pathway reflects the focus of spatial attention. Neuron 60, 709-719.

Siegel, M., and Konig, P. (2003). A functional gamma-band defined by stimulus-dependent synchronization in area 18 of awake behaving cats. J. Neurosci. 23, 4251-4260.

Siegel, M., Kording, K. P., and Konig, P. (2000). Integrating top-down and bottom-up sensory processing by somato-dendritic interactions. J. Comput. Neurosci. 8, 161-173.

Siegel, M., Warden, M. R., and Miller, E. K. (2009). Phase-dependent neuronal coding of objects in short-term memory. Proc. Natl. Acad. Sci. U.S.A. 106, 21341-21346.

Singer, W. (1999). Neuronal synchrony: a versatile code for the definition of relations? Neuron 24, 49-65, 111-125.

Sirota, A., Montgomery, S., Fujisawa, S., Isomura, Y., Zugaro, M., and Buzsaki, G. (2008). Entrainment of neocortical neurons and gamma oscillations by the hippocampal theta rhythm. Neuron 60, 683-697.

Steinmetz, P. N., Roy, A., Fitzgerald, P. J., Hsiao, S. S., Johnson, K. O., and Niebur, E. (2000). Attention modulates synchronized neuronal firing in primate somatosensory cortex. Nature 404, 187-190.

Tiesinga, P.H., Fellous, J.M., Jose, J.V., and Sejnowski, T. J. (2002). Information transfer in entrained cortical neurons. Network 13, 41-66.

Tort, A. B., Komorowski, R. W., Manns, J. R., Kopell, N. J., and Eichenbaum,
H. (2009). Theta-gamma coupling increases during the learning of itemcontext associations. Proc. Natl. Acad. Sci. U.S.A. 106, 20942-20947.

Treisman, A. M., and Gelade, G. (1980). A feature-integration theory of attention. Cogn. Psychol. 12, 97-136.

Usrey, W. M., and Reid, R. C. (1999). Synchronous activity in the visual system. Annu. Rev. Physiol. 61, 435-456.

VanRullen, R., Carlson, T., and Cavanagh, P. (2007). The blinking spotlight of attention. Proc. Natl. Acad. Sci. U.S.A. 104, 19204-19209.

VanRullen, R., Reddy, L., and Koch, C. (2005). Attention-driven discrete sampling of motion perception. Proc. Natl. Acad. Sci. U.S.A. 102, 5291-5296

VanRullen, R., Reddy, L., and Koch, C. (2006). The continuous wagon wheel illusion is associated with changes in electroencephalogram power at approximately 13 Hz. J. Neurosci. 26, 502-507.

Varela, F., Lachaux, J.P., Rodriguez, E., and Martinerie, J. (2001). The brainweb: phase synchronization and large-scale integration. Nat. Rev. 2, 229-239.

von Stein, A., Chiang, C., and Konig, P. (2000). Top-down processing mediated by interareal synchronization. Proc. Natl. Acad. Sci. U. S. A. 97, $14748-14753$

Wang, X. J. (2008). Decision making in recurrent neuronal circuits. Neuron $60,215-234$.

Whittington, M. A., and Traub, R. D. (2003). Interneuron diversity series: inhibitory interneurons and network oscillations in vitro. Trends Neurosci. 26, 676-682.
Wolfe, J. M., Cave, K. R., and Franzel, S. L. (1989). Guided search: an alternative to the feature integration model for visual search. J. Exp. Psychol. 15, 419-433.

Wolfe, J. M., and Horowitz, T. S. (2004). What attributes guide the deployment of visual attention and how do they do it? Nat. Rev. 5, 495-501.

Womelsdorf, T., Fries, P., Mitra, P. P., and Desimone, R. (2006). Gamma-band synchronization in visual cortex predicts speed of change detection. Nature 439, 733-736.

Yantis, S., and Serences, J. T. (2003). Cortical mechanisms of space-based and object-based attentional control. Curr. Opin. Neurobiol. 13, 187-193.

Conflict of Interest Statement: The authors declare that the research was conducted in the absence of any commercial or financial relationships that could be construed as a potential conflict of interest.

Received: 04 June 2010; accepted: 28 September 2010; published online: 02 November 2010.

Citation: Buschman TJ and Miller EK (2010) Shifting the spotlight of attention: evidence for discrete computations in cognition. Front. Hum. Neurosci. 4:194. doi: 10.3389/fnhum.2010.00194

Copyright (C) 2010 Buschman and Miller. This is an open-access article subject to an exclusive license agreement between the authors and the Frontiers Research Foundation, which permits unrestricted use, distribution, and reproduction in any medium, provided the original authors and source are credited. 